University of Wollongong

Research Online

Faculty of Engineering and Information

Faculty of Engineering and Information

Sciences - Papers: Part B

Sciences

2019

Experimental and Self-Consistent Modeling Study of De-twinning in a Twinning-Induced Plasticity Steel

\author{
Ahmed A. Saleh \\ University of Wollongong, asaleh@uow.edu.au \\ Wei Wen \\ Los Alamos National Laboratory \\ Elena V. Pereloma \\ University of Wollongong, elenap@uow.edu.au \\ Scott McCormack \\ University of Wollongong \\ Carlos N. Tome \\ Los Alamos National Laboratory
}

See next page for additional authors

Follow this and additional works at: https://ro.uow.edu.au/eispapers1

Part of the Engineering Commons, and the Science and Technology Studies Commons

Research Online is the open access institutional repository for the University of Wollongong. For further information contact the UOW Library: research-pubs@uow.edu.au 


\title{
Experimental and Self-Consistent Modeling Study of De-twinning in a Twinning- Induced Plasticity Steel
}

\author{
Abstract \\ The effect of compression-tension loading on the microstructure evolution in a fully annealed \\ Fe-24Mn-3Al-2Si-1 Ni-0.06C twinning-induced plasticity steel has been investigated. Electron back- \\ scattering diffraction was used to track a region of interest at true strains of 0 (initial), -0.09 (after \\ forward compression loading), and 0.04 (after reverse tension loading). All deformation twins detected \\ after forward compression loading were found to de-twin upon subsequent reverse tension loading, likely \\ due to the reverse glide of partial dislocations bounding the twins. The reverse loading behavior, including \\ the twinning and de-twinning processes, was successfully simulated using a recently modified \\ dislocation-based hardening model embedded in the visco-plastic self-consistent polycrystal framework, \\ taking into account the dislocation accumulation/annihilation, as well as the twin barrier and back-stress \\ effects. \\ Disciplines \\ Engineering | Science and Technology Studies

\section{Publication Details} \\ Saleh, A. A., Wen, W., Pereloma, E. V., McCormack, S. J., Tome, C. N. \& Gazder, A. A. (2019). Experimental \\ and Self-Consistent Modeling Study of De-twinning in a Twinning-Induced Plasticity Steel. JOM Journal of \\ the Minerals, Metals and Materials Society, 71 (4), 1396-1403.
}

\section{Authors}

Ahmed A. Saleh, Wei Wen, Elena V. Pereloma, Scott McCormack, Carlos N. Tome, and Azdiar Adil Gazder 


\title{
Experimental and self-consistent modeling study of de-twinning in a twinning-induced plasticity steel
}

Ahmed A. Saleh ${ }^{1 *}$, Wei Wen², Elena V. Pereloma ${ }^{1,3}$, Scott J. McCormack ${ }^{1}$, Carlos N. Tomé ${ }^{2}$, Azdiar A. Gazder $^{3}$

${ }^{1}$ School of Mechanical, Materials, Mechatronic and Biomedical Engineering, University of Wollongong, New South Wales 2522, Australia

2Materials Science and Technology Division, Los Alamos National Laboratory, New Mexico 87545, United States

3Electron Microscopy Centre, University of Wollongong, New South Wales 2500, Australia

\begin{abstract}
This work investigates the effect of compression-tension loading on the microstructure evolution in a fully annealed Fe-24Mn-3Al-2Si-1Ni-0.06C twinning-induced plasticity steel. Electron back-scattering diffraction was used to track a region of interest at true strains of 0 (initial), -0.09 (after forward compression loading) and 0.04 (after reverse tension loading). All deformation twins detected after forward compression loading were found to de-twin upon subsequent reverse tension loading, likely due to the reverse glide of partial dislocations bounding the twins. The reverse loading behavior, including the twinning and de-twinning processes, was successfully simulated using a recently modified dislocation-based hardening model embedded in the visco-plastic self-consistent polycrystal framework, taking into account the dislocation accumulation/annihilation, as well as the twin barrier and back-stress effects.
\end{abstract}

Keywords: TWIP steel, twinning, de-twinning, electron back-scattering diffraction (EBSD), VPSC.

*Corresponding author:

Ahmed A. Saleh

School of Mechanical, Materials, Mechatronic and Biomedical Engineering

University of Wollongong, New South Wales 2522, Australia

Phone: +61242213034 Fax: +61242213114 e-mail: asaleh@uow.edu.au 


\section{Introduction}

TWinning Induced Plasticity (TWIP) steels containing 20-30 wt.\% Mn and small additions of $\mathrm{Al}$ and Si are a grade of advanced high strength steels with a unique combination of high strength (600-1000 $\mathrm{MPa}$ ) and large ductility ( $>50 \%$ ) [1]. TWIP steels comprise a stable face centered cubic (fcc) austenite phase with low stacking fault energy (SFE $=18-40 \mathrm{~mJ} \mathrm{~m}^{-2}$ ) that instigates twinning along with dislocation glide during room temperature deformation. Moreover, this low SFE promotes the dissociation of perfect dislocations gliding on the $\{111\}$ plane in the $\langle 110\rangle$ direction into $\langle 112\rangle$ Shockley partials bounding stacking faults [2]. A deformation twin forms when the dissociated partials diverge from each other to an infinite separation distance [2].

While the monotonic loading behavior of TWIP steel has been the focus of numerous experimental and modeling studies (see for example [3-4] and the references therein), investigations of its cyclic (reverse) loading behavior remain relatively limited. In this regard, an Fe-24Mn-3Al-2Si-1 Ni-0.06C (wt.\%) TWIP steel subjected to $\pm 1 \%$ cyclic (tension-compression) loading was recently investigated using a combination of in-situ neutron diffraction and an elasto-plastic self-consistent model [5-6]. The modeling work in Ref. [6] suggested that de-twinning could be taking place upon load reversal due to the reversibility of slip along the $\langle 112\rangle$ direction. To verify the occurrence of de-twinning (i.e., the disappearance of twinned regions as the twins are reoriented back to their parent grains), electron backscattering diffraction (EBSD) was applied in a follow-up study to track microstructure evolution in a region of interest during interrupted reverse (tension-compression) loading of the same TWIP steel [7]. All deformation twins observed after forward tension loading to a true strain of 0.128 were found to detwin upon subsequent reverse compression loading back to a true strain of 0.031 . Consequently, the former study provided the first direct experimental evidence of de-twinning during load reversal in TWIP steel, in particular, and low SFE coarse-grained (micron-sized) polycrystalline fcc materials, in general.

Along with the experimental observation of de-twinning in the above study [7], the tensioncompression loading behavior was successfully simulated using a dislocation-based hardening model embedded in the visco-plastic self-consistent (VPSC) polycrystal framework [8-9]. The hardening model is based on the Rauch-Gracio-Barlat-Vincze (RGBV) [10] continuum plasticity model developed to simulate the mechanical response under strain path changes; building on the concept that a subset of dislocations generated during loading in one direction can reverse their glide direction and progressively recombine upon load reversal. Kitayama et al. [11] reformulated the RGBV model for a crystallographic framework to explicitly account for the changes in strain path by tracking the shear reversal on each slip system within every grain. Thereafter, Wen et al. extended the crystallographic RGBV model to predict the mechanical response associated with tension reloading of AZ31 Mg alloy [12] and cyclic shear tests of low-carbon steel [13]. The model was further modified in order to account for the processes of twinning and de-twinning, as well as the back stress and twin barrier effects under tension-compression loading of TWIP steel [7]. 
The present study aims to verify the occurrence of de-twinning in the same Fe-24Mn-3Al-2Si-1 Ni0.06C TWIP steel discussed above, when subjected to a different loading sequence; namely, compression-tension. To this end, EBSD is applied to track microstructure evolution in a region of interest during interrupted compression-tension loading. The modified RGBV-VPSC model [7] is also used in the present work to further validate our formulation of the twinning back-stress and the twin barrier effect introduced to capture the processes of twinning and de-twinning (or twin lamellae generation and annihilation) during load reversal.

\section{Experimental procedure}

An as-cast slab of Fe-24Mn-3Al-2Si-1Ni-0.06C wt.\% TWIP steel was homogenized at $1100{ }^{\circ} \mathrm{C}$ for 2 $\mathrm{h}$, hot rolled to $52 \%$ thickness reduction at the same temperature then cold rolled to $42 \%$ thickness reduction. A dog-bone shaped sample (Fig. 1) of $4 \mathrm{~mm}$ gage length, $4 \mathrm{~mm}$ width and $4 \mathrm{~mm}$ thickness was wire-cut from the middle of the cold rolled strip such that the gauge length and width were parallel to the rolling and normal directions, respectively. The dimensions of the dog-bone shaped sample were deliberately chosen to avoid buckling during compression.

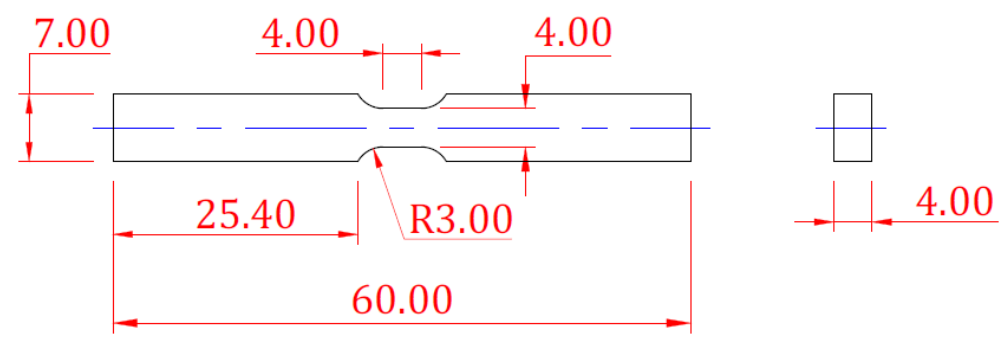

Figure 1. Dimensions (in $\mathrm{mm}$ ) of the dog-bone sample used for forward compression - reverse tension loading.

In order to obtain a fully recrystallized microstructure, the dog-bone sample was annealed at 850 ${ }^{\circ} \mathrm{C}$, including $240 \mathrm{~s}$ of heating to a stable temperature and $300 \mathrm{~s}$ of soaking time followed by immediate water quenching. Thereafter, the sample was mechanically polished up to the colloidal silica stage. Uniaxial compression-tension testing was undertaken on a servo-hydraulic universal machine operating in speed control mode at $0.005 \mathrm{~mm} / \mathrm{s}$. The test was interrupted at a true strain of -0.09 in compression followed by reverse loading in tension back to a true strain of 0.04 .

EBSD was conducted on a JEOL-JSM7001F field emission gun-scanning electron microscope fitted with a Nordlys-II(S) camera and the Oxford Instruments AZtec software suite; operating at $15 \mathrm{kV}$ accelerating voltage, $\sim 5 \mathrm{nA}$ probe current and $15 \mathrm{~mm}$ working distance. EBSD mapping was conducted on a selected area of interest in the middle of the gage length at true strains of 0 (initial), -0.09 (after forward compression loading, C) and 0.04 (after reverse tension loading, CT), using a step size of $0.1 \mu \mathrm{m}$. 
Post-processing of the EBSD maps was carried out using the Oxford Instruments Channel-5 software package. In all maps, (sub)grain structures were defined by a minimum of three pixels. Lowangle grain boundaries (LAGBs) and high-angle grain boundaries (HAGBs) comprise misorientations between $2^{\circ} \leq \theta<15^{\circ}$ and with $\theta \geq 15^{\circ}$, respectively. The twin boundaries (TBs) consist of first order $\left(\Sigma 3=60^{\circ}\langle 111\rangle\right)$ and second order $\left(\Sigma 9=38.9^{\circ}\langle 110\rangle\right)$ TBs. The maximum deviation of the misorientation angle $(\Delta \theta)$ from the exact axis-angle relationship was defined using the Palumbo-Aust criterion (i.e., $\Delta \theta \leq 15^{\circ} \Sigma^{-5 / 6}$ ] [14], resulting in tolerance limits of $6^{\circ}$ for $\Sigma 3$ and $2.4^{\circ}$ for $\Sigma 9$.

\section{Visco-plastic self-consistent modelling}

Since the RGBV-VPSC model detailed in Ref. [7] is also applied in this study, the model formulation is not reproduced here in order to ensure brevity in the text. Briefly speaking, the model is based on the RGBV-VPSC framework [11-13] and has been modified to take into account the twinning barrier effect (as deformation twins act as strong obstacles to dislocation glide) and the occurrence of de-twinning under strain path change [7]. While the inclusion formalism of the VPSC model inherently accounts for the intergranular stresses effect, the intragranular stresses are captured via empirical back stress equations introduced for both slip and twinning systems. The back stress contribution to the hardening of slip systems is linked to reversible dislocations, whereas the back stress associated with the formation of twins is due to the large localized shear introduced in the grain. The effect of the generation/annihilation of deformation twins on hardening is formulated via the changes in the dislocation mean free path.

The VPSC model does not account for elasticity, therefore it is not possible to fit the initial elastic loading regime or the elastic unloading upon load reversal. In order to rigorously assess the model performance, the same hardening parameters used previously to simulate the tension-compression loading case [7] were used to simulate the present compression-tension experiment (see Tables 1 and 2 in Ref. [7]). The fully annealed material exhibits a weak texture. The initial texture input comprised 5000 discrete orientations calculated from the fully annealed texture measured via X-ray diffraction. Similar to Ref. [7], the intermediate grain-matrix interaction scheme $n^{\text {eff }}=10$ was used for the present simulations. The plastic deformation modes introduced into the VPSC model included the $\{111\}\langle 110\rangle$ slip systems and the $\{111\}\langle 112\rangle$ twinning systems. It is noted that the activation and growth of twinning in the parent grain follows the original VPSC model [8-9] in that the activation of twinning in the negative direction is prevented by enforcing the condition $\dot{\gamma}^{t}$ (twin shear rate) $\geq 0$. However, since de-twinning corresponds to $\dot{\gamma}^{t} \geq 0$ in the twinned volume and $\dot{\gamma}^{t}<0$ in the parent, the resolved shear on the twin plane is calculated using the stress of the parent and de-twinning is activated when $\dot{\gamma}^{t}<0$ in the parent. This procedure is stopped if/when all twins de-twin and have recovered their parent grain orientation. 


\section{Results and discussion}

\subsection{Experimental observations}

Fig. 2 shows the experimental true stress versus strain curve during forward compression loading (C) up to a true strain of -0.09 followed by reverse tension loading (CT) back to a true strain of 0.04 . Fig. 3 a depicts the initial fully annealed microstructure, which is characterized by equiaxed grains with an average grain size of $6.6 \pm 4.9 \mu \mathrm{m}$ (without considering twin boundaries) and a high fraction of annealing twin boundaries ( $\sim 44 \%$ of the total boundary length fraction). Fig. $3 \mathrm{~b}$ shows the same mapped area after forward compression loading (C) to a true strain of -0.09. As indicated by the yellow ovals in Fig. $3 \mathrm{~b}$, deformation twins were found in the form of single lines or parallel packets and expectedly tended to evolve in grains with orientations close to $\langle 100\rangle$ parallel to the compression axis. This preference for twin formation in the $\langle 100\rangle$-oriented grains under compression loading is in accordance with Schmid's law, as they exhibit a higher Schmid factor for twinning than slip.

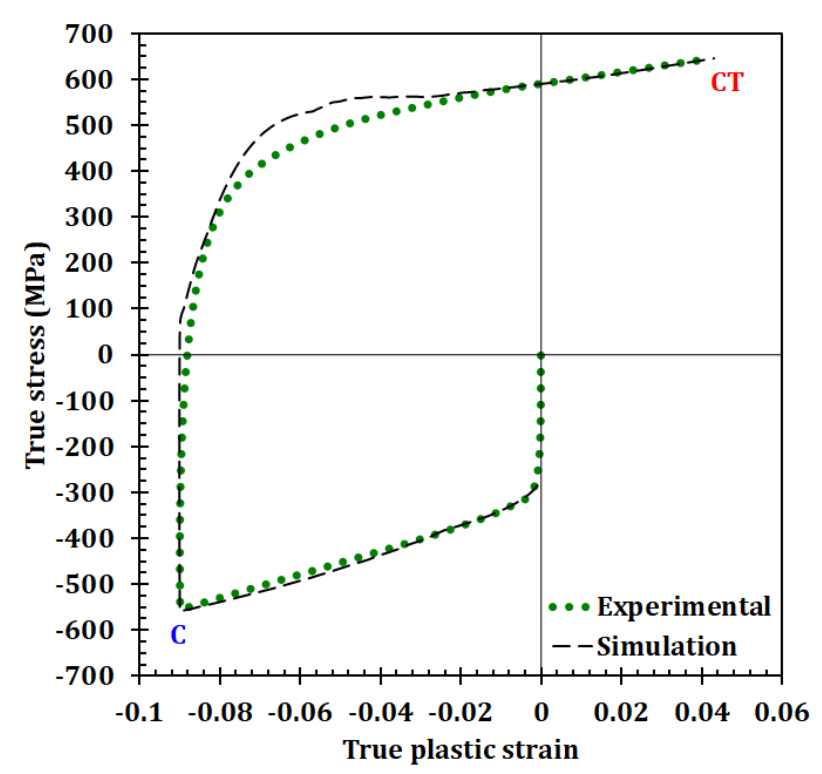

Figure 2. The experimental and VPSC simulated true stress vs. true plastic strain during forward compression (C) - reverse tension (CT) loading. 
(a)

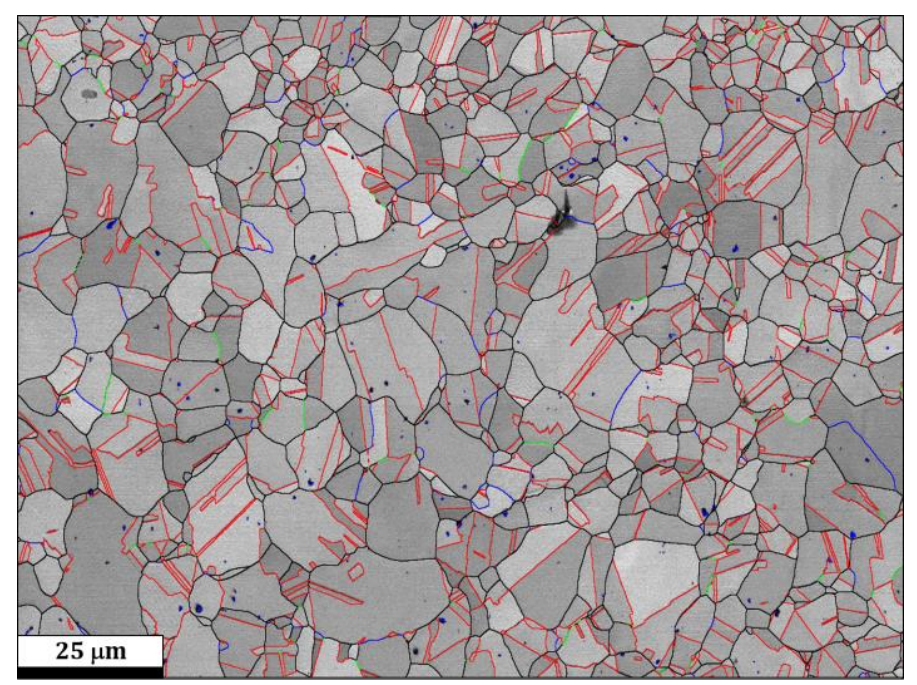

(b)
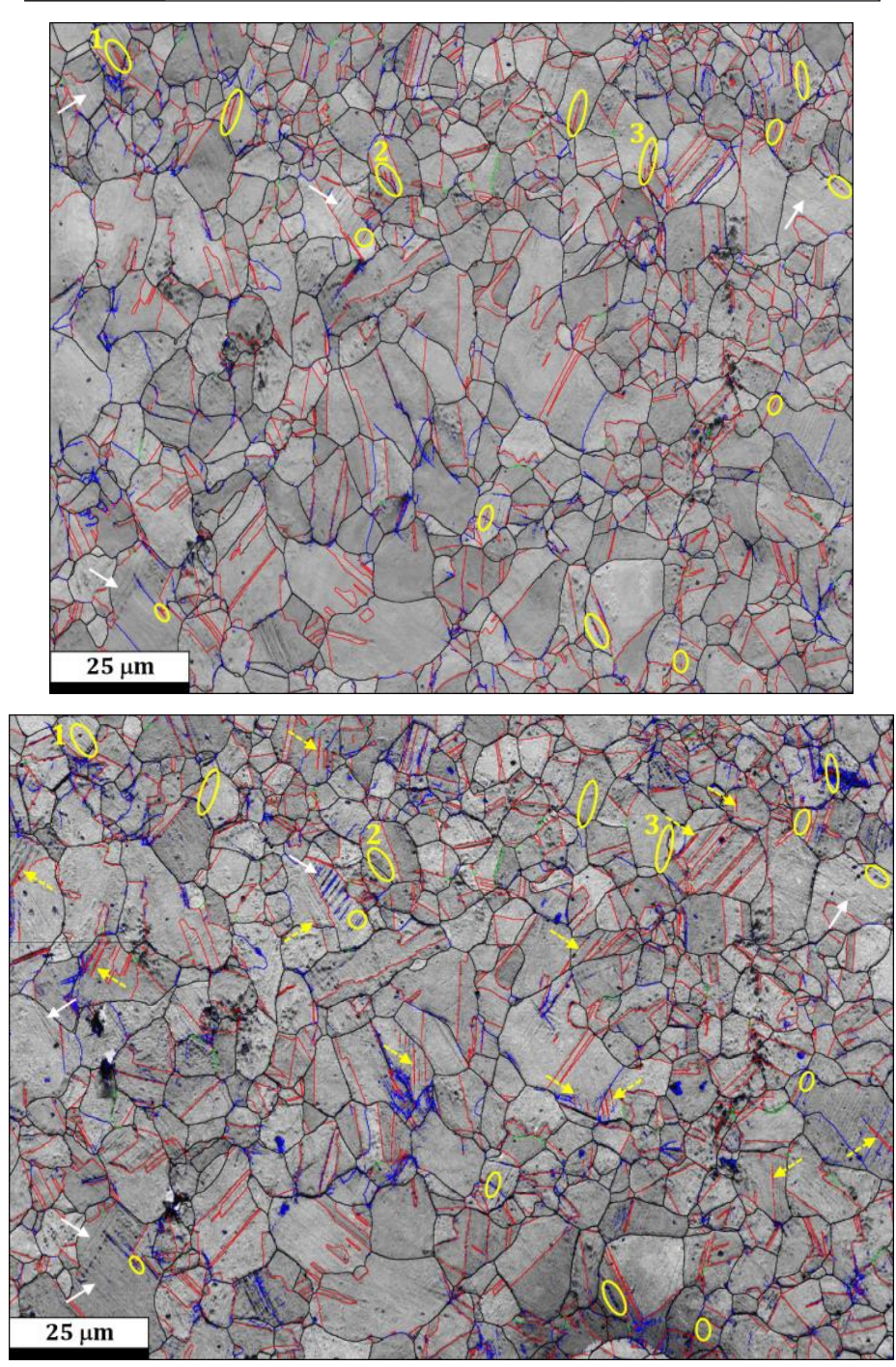

(c)

Figure 3. Band contrast maps at true strains of (a) 0 , initial, (b) -0.09 , after forward compression loading (C) and (c) 0.04, after reverse tension loading (CT). LAGBs = blue, HAGBs = black, $\Sigma 3$ $\left(60^{\circ}\langle 111\rangle\right)=$ red and $\Sigma 9\left(38.9^{\circ}\langle 110\rangle\right)=$ green. The yellow ellipses indicate regions where twinning and de-twinning were observed. The loading direction is horizontal. 
It is also noted that the amount of deformation twins formed in this study under forward compression loading is relatively less than the forward tension loading case investigated previously [7]. This is ascribed to the directionality of twinning which, when combined with texture, leads to different subsets of grains having high Schmid factors for twinning when the sample is subjected to tension or compression [15]. For the present fully annealed TWIP steel with its initially weak texture, it is less likely to activate twinning during compression compared to tension [5]. This can be theoretically rationalized as follows. For randomly oriented fcc polycrystals deforming only by $\{111\}\langle 112\rangle$ twinning, Hosford and Allen [15] used Bishop and Hill- type analysis to show that the yield stress in uniaxial compression should be $28 \%$ higher than in uniaxial tension. Using Taylor-type analysis, Chin et al. [16] also reported a $25 \%$ difference between the compressive and tensile yield stresses. Additionally, Hosford and Allen [15] pointed out that the same trend persists when both slip and twinning are considered in the calculations, but the differences between the compressive and tensile yield stresses would be relatively smaller. In line with the former idea, during our previous EPSC simulations of the present TWIP steel (accounting for both slip and twinning and using the same initial weak texture as an input), the stress required to activate twinning under compression was predicted to be $\sim 20 \%$ higher than that under tension [5].

Additionally, some in-grain striations were also observed after forward compression loading as indicated by the white arrows in the band contrast map shown in Fig. 3b. These striations can be associated with fine deformation twins (with thicknesses in the order of tens of nanometers) that are not crystallographically detected via EBSD due to spatial resolution limitations [4]. As the activity of deformation twinning increases, these fine twins stack into relatively thick bundles such that they are then crystallographically detected by EBSD. Alternatively, other studies have suggested that some of these in-grain striations could be stacking faults that serve as precursors to deformation twinning [17].

Subsequent reverse tension loading (CT) back to a true strain of 0.04 resulted in the removal of all deformation twins previously formed during compression (compare the yellow ovals in Figs. 3b and 3c), along with the formation of new twins and in-grain striations ${ }^{a}$ (refer to the yellow and white arrows in Fig. 3c, respectively). The former observation verifies the occurrence of de-twinning during compression-tension loading of TWIP steel and corroborates our earlier tension-compression loading results [7].

Fig. 4 shows representative inverse pole figure (IPF) maps of grains 1, 2 and 3 marked in Fig. 3b after forward compression (Figs. 4a, 4d and 4g) and reverse tension loading (Figs. 4b, 4e and 4h). The orientations of grains 1, 2 and 3 are given in the caption of Fig. 4. The misorientation profiles along the black dashed lines show clear evidence of twinning and de-twinning after forward compression and reverse tension loading in all three grains (Figs. 4c, 4f and 4i).

\footnotetext{
a Some of the in-grain striations formed during compression disappeared upon reverse tension loading, while some remain and could still be seen in Fig. 3(c). However, due to the uncertainty of the nature of these in-grain striations, any further discussion of their evolution upon reverse loading would be highly speculative.
} 


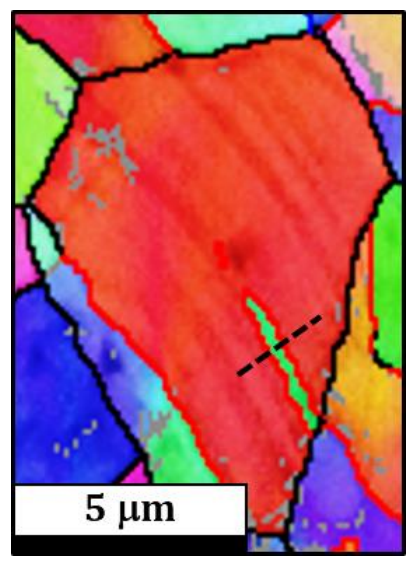

(a)

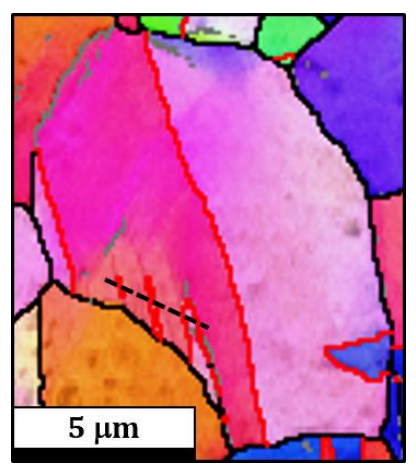

(d)

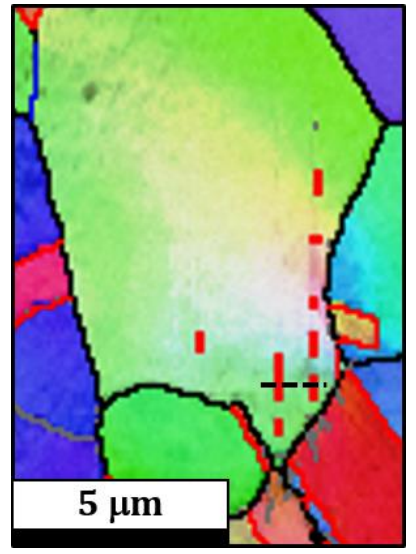

(g)

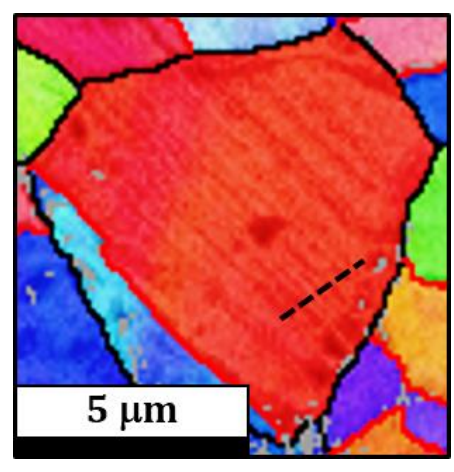

(b)

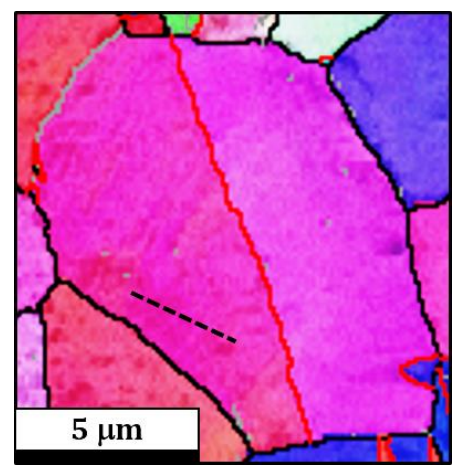

(e)

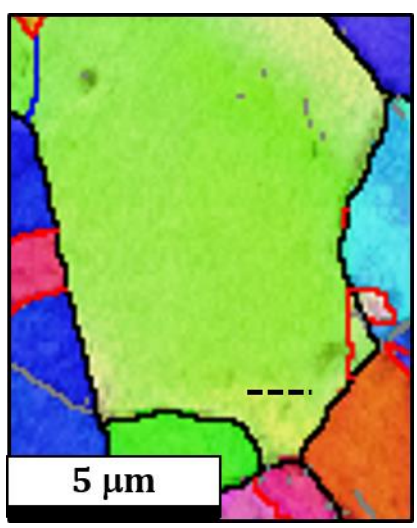

(h)

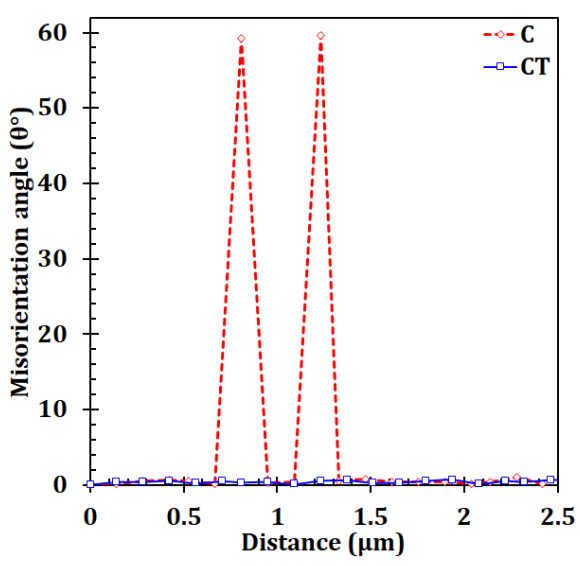

(c)

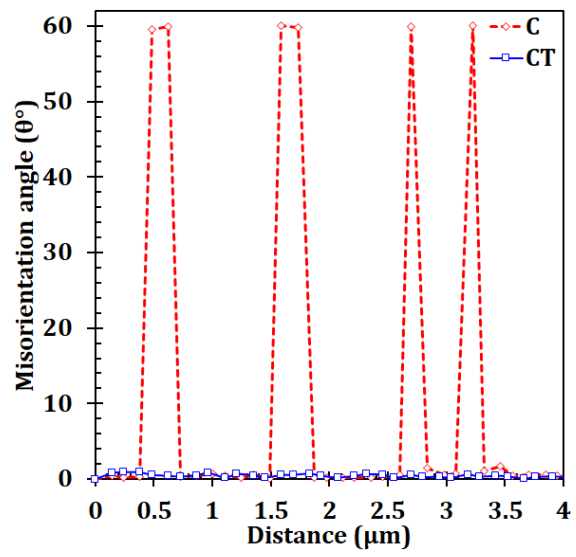

(f)

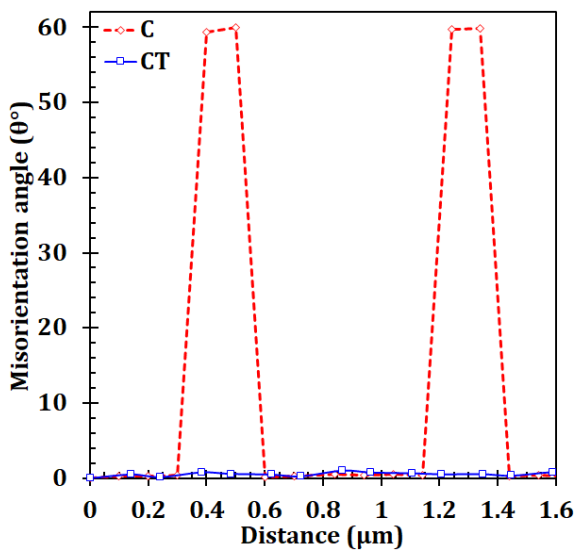

(i)

Figure 4. Representative inverse pole figure maps of grains 1, 2 and 3 marked in Fig. $3 b$ after $(a, d, g)$ forward compression and $(b, e, h)$ reverse tension loading. (c, f, i) Misorientation profiles along the black dashed lines evidence twinning and de-twinning after forward compression (C) and reverse tension (CT) loading. The orientations of grains 1, 2 and 3 are indicated on the given IPF. Grain $1=(001)[0 \overline{1} 0]$, grain $2=(011)[4 \overline{1} 1]$ and grain $3=(113)$ [ $\overline{7} 41]$. LAGBs $=$ grey,

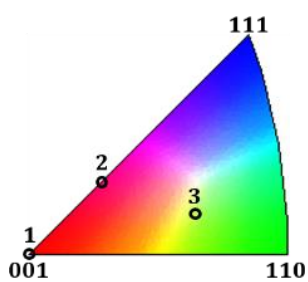
HAGBs $=$ black and $\Sigma 3\left(60^{\circ}\langle 111\rangle\right)=$ red. The loading direction is horizontal. 
In the widely investigated hcp materials [18-22], de-twinning upon load reversal is associated with the reverse glide of twinning dislocations present at the twin interface. In hcp materials, de-twinning is more likely to occur than the activation of new slip or twinning systems, due to the limited number of slip systems available. In the limited experimental and modeling studies available on cubic materials (such as the experiments on $\mathrm{Cu}-8.5 \mathrm{Al}$ fcc single crystals [23] and the phase field simulations of a polycrystalline fcc metal [24]), de-twinning was ascribed to the partials bounding a twin being able to glide in the reverse direction upon unloading, leading to the reorientation of the twinned region back to its parent grain orientation. Analogous to the restricted slip effect in hcp materials, de-twining in the $\mathrm{Cu}$ $8.5 \mathrm{Al}$ fcc single crystals was promoted by the restriction imposed by the existing deformation twins on the number of operative slip planes. This restriction likely causes the partials bounding the twin to reverse direction, instead of activating another twin system, and results in de-twinning [23].

\subsection{VPSC model predictions}

The simulated stress-strain curve is compared with its experimental counterpart in Fig. 2. As mentioned previously, since the VPSC model does not account for elasticity, the elastic part was removed from the experimental stress-strain data in order to allow for a direct comparison with the simulated curve. It is also reiterated that no optimization of the hardening parameters was undertaken in the present work. The same parameters used previously to simulate the tension-compression loading case (Tables 1 and 2 in Ref. [7]) were used here. While the stress-strain curve is well captured during forward compression loading, the reload transition is slightly over-predicted during the initial stages of reverse tension loading (Fig. 2). The reason is to be found in the dislocation annihilation mechanism; it starts a

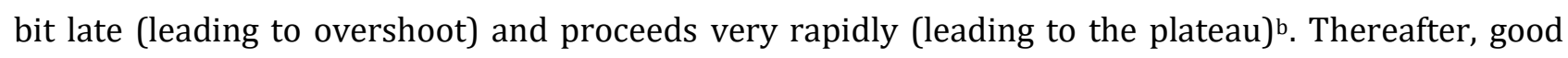
agreement between the experimental and simulated curves is obtained with increasing reverse tensile strain.

Fig. 5a shows the VPSC model predictions of the relative slip, twinning and de-twinning activities as a function of the accumulated true strain. During forward compression loading, higher slip activity is obtained compared to twinning such that a gradual decline in twinning activity is noted with increasing compressive strain. During the initial stages of reverse tension loading, deformation is accommodated only via slip as the strong back-stress developed during forward compression loading promotes slip upon reverse loading. In other words, the slip systems activated during forward compression loading are easier to activate (in the opposite slip direction) upon load reversal, as their generated back stress adds to the resolved shear stress component of the applied tensile stress.

\footnotetext{
b The over-prediction during the initial stage of reverse tension loading could be improved by adjusting the dislocation recombination parameters (see Section 3.1 in Ref. [7]), which dictates the dislocation annihilation rate (and hence the hardening), as the reversible dislocations generated during forward loading progressively recombine upon load reversal. However, such adjustment would defeat our purpose of using the same model parameters as in Ref. [7] and therefore it was not undertaken.
} 
The subsequent decrease in relative slip activity is accompanied by simultaneous increases in both, twinning and de-twinning activities. In this regard, de-twinning is facilitated by the back-stress associated with the previously activated twinning systems as their opposite systems become softer. The observed gradual decrease in de-twinning activity with further reverse tension loading (Fig. 5a) is due to the removal of the twins that formed during forward compression loading.

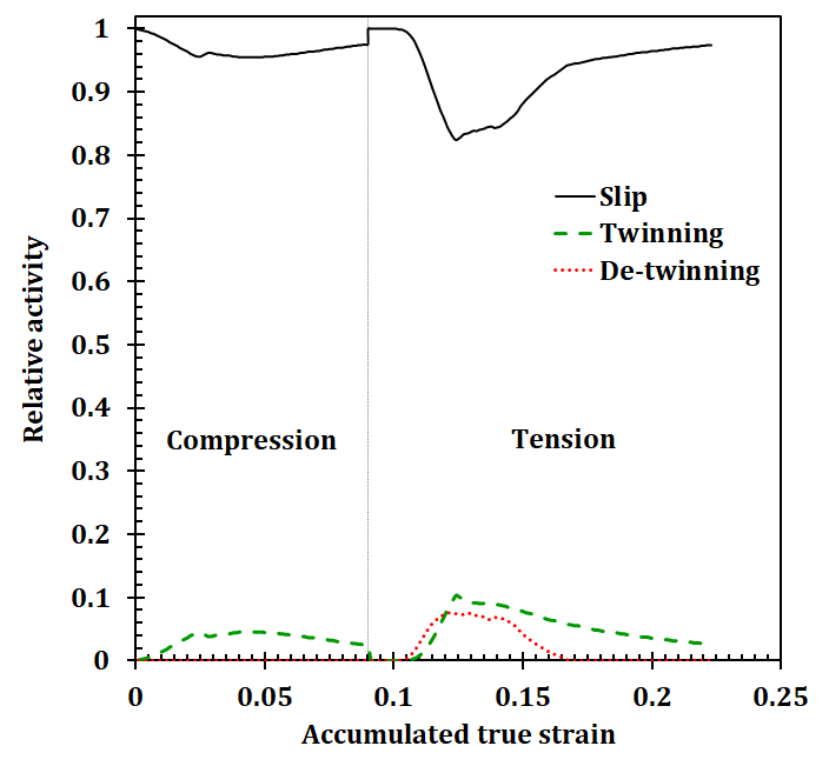

(a)

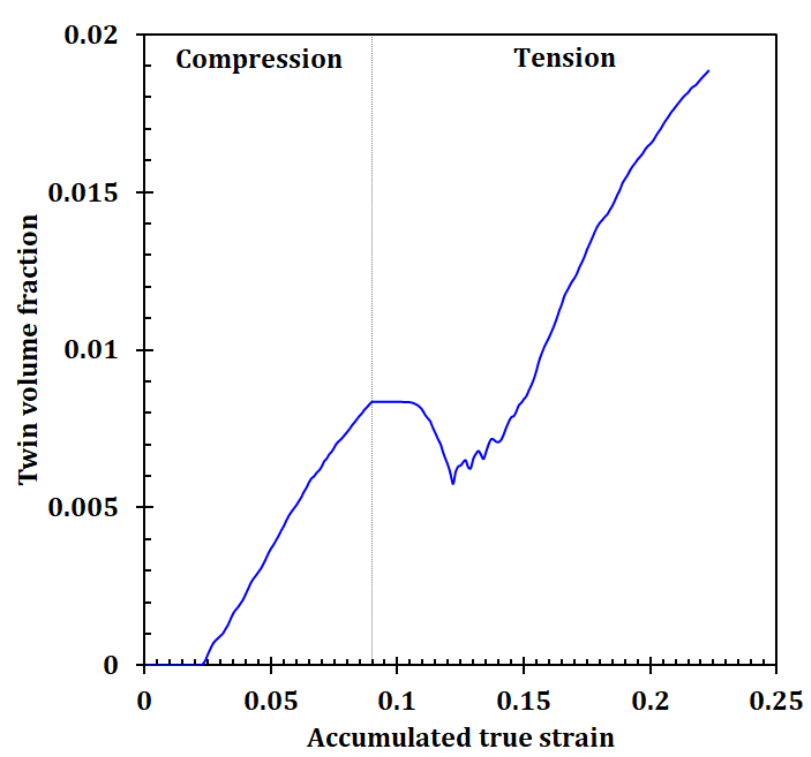

(b)

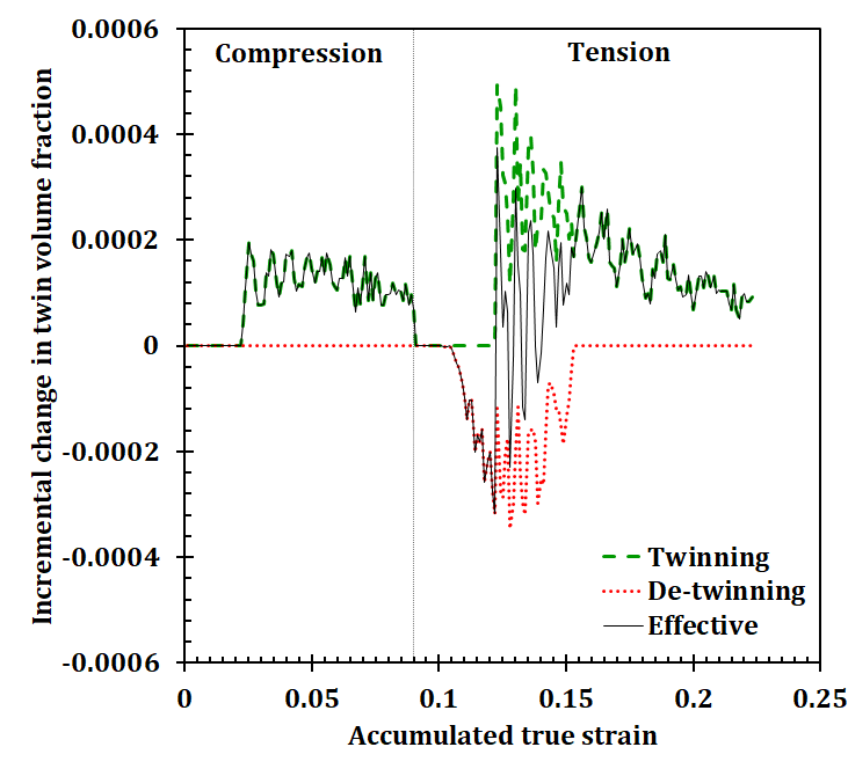

(c)

Figure 5. VPSC model predictions of the (a) relative slip, twinning and de-twinning activities, (b) volume fraction of twins and (c) change in the twin volume fraction due to twinning and de-twinning activities. 
In accordance with the discussion in Section 4.1, two additional observations linked to the directionality of twinning and Schmid factor considerations [15] are noted in Fig. 5a. (i) The relative twinning activity during reverse tension loading is higher than during forward compression loading. (ii) More interestingly, the nearly equal twinning and de-twinning activities predicted during the initial stages of reverse tension loading is different from the forward tension - reverse compression case in Ref. [7]. Therein de-twinning was predicted to be dominant during the early stages of reverse compression loading; with only limited twinning activity during compression. It follows that in the present case, as de-twinning is underway upon load reversal from compression to tension, new twins simultaneously form in other favorably oriented grains. The new twins observed in Fig. 3c (indicated by yellow arrows) clearly corroborates the above model predictions during reverse tension loading.

The predicted twin volume fraction shown in Fig. $5 \mathrm{~b}$ follows the above discussion on relative systems activity. Less than $1 \%$ twin volume fraction is predicted at the end of forward compression loading followed by a short plateauing during the initial stages of reverse tension loading when only slip is active. The subsequent decline in the twin volume fraction signifies the dominance of de-twinning activity, until twinning takes over and the volume fraction gradually increases to $\sim 2 \%$ at the end of reverse tension loading. It is highlighted that the limited twin volume fraction predicted here is typically reported for low SFE materials in general and TWIP steel in particular [4-5, 25-26].

Fig. 5c depicts the individual contributions of twinning (dashed green line) and de-twinning (dotted red line) to the change in twin volume fraction, with the effective (solid black line) being the difference between the two mechanisms. While the effective and twinning lines match each other exactly during forward compression loading, the effective and de-twinning lines follow one another when detwinning is dominant during the early stages of reverse tension loading. Thereafter, the effective line is in-between the twinning and de-twinning lines while the two mechanisms are concurrent. When detwinning is completed (returns to zero activity), the effective line follows the twinning line again.

Lastly, while it is beyond the scope of the present work, it would be highly desirable to apply the current RGBV-VPSC model to forward-reverse loading paths involving higher strains (for example, forward-reverse torsion or shear loading) as this would further validate the input parameters for the model. Subsequently, the model can be utilized to simulate the complex strain path changes that take place during industrial forming processes. With regard to TWIP steels, it is highlighted that the reverse glide of the partial dislocations and the associated de-twinning process likely contribute to the pronounced Bauschinger effect (or early yielding during unloading) typically observed upon load reversal [5-6, 27].

\section{Conclusions}

Tracking the microstructure evolution of a selected area of interest via EBSD mapping verified the occurrence of de-twinning during interrupted compression-tension loading of a TWIP steel. Detwinning during load reversal is likely due to the partial dislocations bounding the deformation twin 
being able to glide in the reverse direction resulting in the reorientation of the twin back to its parent grain orientation. The successful simulation of the reverse loading behavior further validates the RGBVVPSC model and supports our formulation and implementation of the twin barrier and back-stress effects that account for the processes of twinning and de-twinning during load reversal.

\section{Acknowledgments}

This work was funded by the Australian Research Council - Discovery Projects DP130101882 and DP170100836. The authors thank Prof. D.B. Santos (UFMG, Brazil) for providing the source material and Mr. Heiko Tysar (BlueScope Steel Limited) for his help with the mechanical testing. CNT was fully supported by the U.S. DOE project FWP 06SCPE401 under U.S. DOE contract W-7405-ENG-36. The JEOL JSM-7001F FEG-SEM was funded by the Australian Research Council - Linkage Infrastructure, Equipment and Facilities grant LE0882613.

\section{References}

[1] O. Grassel, L. Kruger, G. Frommeyer and L.W. Meyer, Int. J. Plasticity, 16, 1391 (2000).

[2] T.S. Byun, Acta Mater., 51, 3063 (2003).

[3] D. Barbier, N. Grey, S. Allain, N. Bozzolo and M. Humbert, Mater. Sci. Eng. A, 500, 196 (2009). [4] A.A. Saleh, E.V. Pereloma and A.A. Gazder, Acta Mater., 61, 2671 (2013).

[5] A.A. Saleh, E.V. Pereloma, B. Clausen, D.W. Brown, C.N. Tomé and A.A. Gazder, Acta Mater., 61, 5247 (2013).

[6] A.A. Saleh, B. Clausen, D.W. Brown, E.V. Pereloma, C.H.J. Davies, C.N. Tomé and A.A. Gazder, Mater. Lett., 182, 294 (2016).

[7] S.J. McCormack, W. Wen, E.V. Pereloma, C.N. Tomé, A.A. Gazder and A.A. Saleh, Acta Mater., 156, 172 (2018).

[8] R.A. Lebensohn and C.N. Tomé, Acta Metall. Mater., 41, 2611 (1993).

[9] R.A. Lebensohn, C.N. Tomé and P.P. CastaÑeda, Philos. Mag., 87, 4287 (2007).

[10] E.F. Rauch, J.J. Gracio, F. Barlat and G. Vincze, Modell. Simul. Mater. Sci. Eng., 19, 035009 (2011).

[11] K. Kitayama, C.N. Tomé, E.F. Rauch, J.J. Gracio and F. Barlat, Int. J. Plasticity, 46, 54 (2013).

[12] W. Wen, M. Borodachenkova, C.N. Tomé, G. Vincze, E.F. Rauch, F. Barlat and J.J. Grácio, Int. J. Plasticity, 73, 171 (2015).

[13] W. Wen, M. Borodachenkova, C.N. Tomé, G. Vincze, E.F. Rauch, F. Barlat and J.J. Grácio, Acta Mater., 111, 305 (2016).

[14] G. Palumbo and K. Aust, Acta Metall. Mater., 38, 2343 (1990).

[15] W.F. Hosford and T.J. Allen, Metall. Trans., 4, 1424 (1973).

[16] G.Y. Chin, W.L. Mammel and M.T. Dolan, Transactions of the Metallurgical Society of AlME, 245, 383 (1969).

[17] A.A. Gazder, A.A. Saleh, A.G. Kostryzhev and E.V. Pereloma, Mater. Today: Proc., 2, S647 (2015).

[18] G. Proust, G.C. Kaschner, I.J. Beyerlein, B. Clausen, D.W. Brown, R.J. McCabe and C.N. Tomé, Exp. Mech., 50, 125 (2010).

[19] D. Sarker and D.L. Chen, Scripta Mater., 67, 165 (2012).

[20] H. Wang, P.D. Wu, C.N. Tomé and J. Wang, Mater. Sci. Eng. A, 555, 93 (2012).

[21] H. Wang, P.D. Wu, J. Wang and C.N. Tomé, Int. J. Plasticity, 49, 36 (2013). 
[22] L. Wu, S.R. Agnew, D.W. Brown, G.M. Stoica, B. Clausen, A. Jain, D.E. Fielden and P.K. Liaw, Acta Mater., 56, 3699 (2008).

[23] M.S. Szczerba, S. Kopacz and M.J. Szczerba, Acta Mater., 60, 6413 (2012).

[24] S. Hu, C.H. Jr and L. Chen, Acta Mater., 6554 (2010).

[25] T. Leffers and R.K. Ray, Prog. Mater Sci., 54351 (2009).

[26] A.A. Saleh, E.V. Pereloma, B. Clausen, D.W. Brown, C.N. Tomé and A.A. Gazder, Mater. Sci. Eng. A, 589, 66 (2014).

[27] O. Bouaziz, S. Allain and C. Scott, Scripta Mater., 58, 484 (2008). 